\title{
Bilateral cerebellar haemorrhage after lumbar drain placement in a case of primary intraventricular bleed- complication revisited
}

\begin{abstract}
Background and Importance: Lumbar drain placement and diversion of cerebrospinal fluid is a therapeutic procedure, often done bedside to accentuate wound healing in case of dural transgression following cranial or spinal procedures. Indications include cases of open head injuries with Cerebrospinal fluid (CSF) leak and for re-establishment of CSF flow in primary intraventricular bleed with prolonged periods of External ventricular drain (EVD) placement. Complications include central herniation as a result of over drainage of spinal fluid, low-pressure headache and, in some cases profound dyselectrolytemia and nausea and vomiting.

Clinical Presentation: We illustrate a complication, which presented to us, in a 42-year old gentleman with primary intraventricular bleed with EVD in situ. Lumbar drain placement was planned to re-establish CSF flow and for EVD removal, however, once spinal fluid was drained patient deteriorated neurologically. He developed a tight posterior fossa secondary to tonsillar herniation with vermian foliar haemorrhage and had to undergo emergency surgical decompression as a salvage procedure.

Conclusions: Herniation following CSF diversion is a known complication, however, they are less often seen after a lumbar drain placement. Early clinical suspicion and posterior fossa decompression can prevent, the otherwise, lethal outcome in such patients. Here we discuss the complications that ensued and the management thereafter in this clinical vignette.
\end{abstract}

Keywords: lumbar drain, Cerebellar bleed, Herniation, Foliar haemorrhage
Volume 3 Issue 4 - 2015

\section{Rajesh Parameshwaran Nair,' Vinod Kumar,' Sibhi Ganapathy, ${ }^{3}$ Ajay Hegde ${ }^{4}$ \\ 'Senior Registrar of Neurosurgery, Manipal University, India ${ }^{2}$ Associate Professor of Neurosurgery, Manipal University, India Senior Registrar of Neurosurgery, Manipal University, India ${ }^{4}$ Registrar of Neurosurgery, Manipal University, India}

Correspondence: Dr. Rajesh P Nair, MS, MCh, Registrar, Department of Neuroosurgery, Kasturba Medical College, Manipal, Manipal University, India, Email rajeshnair39@yahoo.com

Received: October 27, 2015 | Published: November 25, 2015

\section{Case report}

A 42-year old patient presented to the triage with high-grade fever and protracted vomiting of 3 days duration. He also complained of headache, which was holocranial in nature. Following this, the patient attenders noticed progressive deterioration in the patient's sensorium with associated blurring of vision. He was immediately brought to the triage and on general examination, blood pressure was recorded at $170 / 110 \mathrm{~mm} \mathrm{Hg}$. He was opening eyes to call and was obeying simple commands, however he seemed disoriented. Vision and other cranial nerves could not be assessed and his pupils were bilaterally equal and reacting briskly. Preliminary Computed tomographic scan (CT Scan) of the brain showed primary intraventricular bleed, within the left lateral ventricle and tracking down into the third ventricle, with early hydrocephalus (Figure 1A). External ventricular drainage with instillation of thrombolytics (30,000 IU of streptokinase) was done. He was not started on systemic anticoagulant for deep venous thrombosis (DVT) prophylaxis. The patient showed incremental neurological improvement within the next 24-48 hours. Repeat scans after 4 days post EVD, showed significant decrease in the ventricular size, however the intraventircular haematoma persisted (Figure 1B). $\mathrm{CT}$ angiography was negative for arteriovenous malformations and aneurysms. Patient showed significant neurological improvement and was planned for lumbar drain placement to re-establish normal CSF flow. Immediately after the lumbar drain placement patient deteriorated neurologically and developed pupillary asymmetry. Repeat CT scan of the brain showed bilateral symmetrical cerebellar folial bleed with a tight posterior fossa (Figure C \& D). Lumbar drain was blocked and he was intubated and taken up for sub-occipital craniectomy and evacuation of bilateral cerebellar haematoma. Postoperatively, he was sedated and ventilated for the first 48 hours and showed marginal neurological improvement thereafter. 72 hours after surgery, the patient remained neurologically obtunded and on postoperative day 5 , the attenders deferred further treatment due to financial constraints and discharged the patient against medical advice.
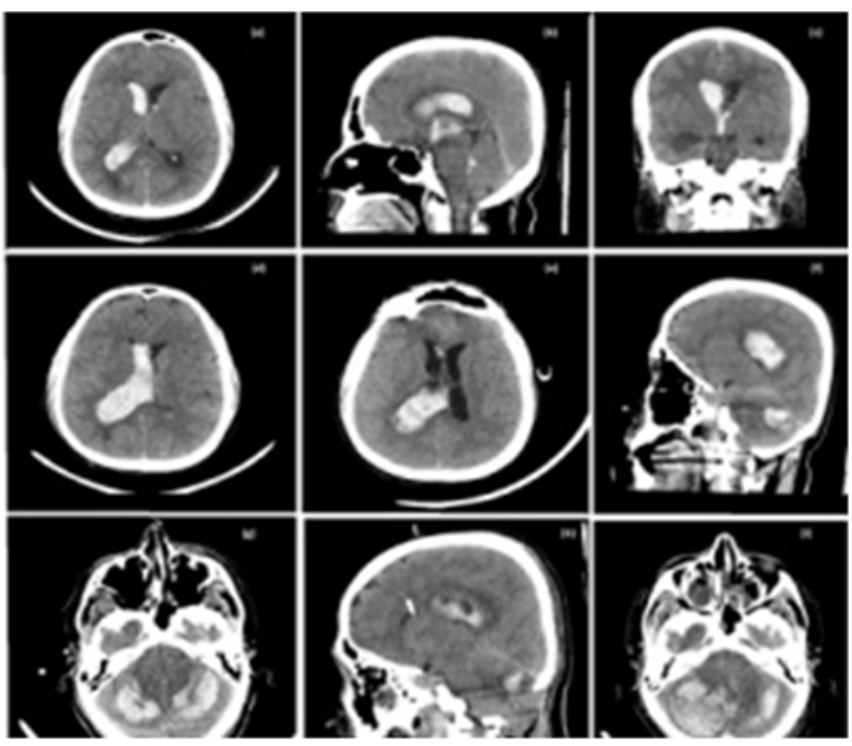

Figure I Darkening of the skin of the forehead with brittle, matted hair.

\section{Discussion}

CSF diversion is a widely accepted and used intervention in cases of CSF leaks of traumatic, spontaneous and iatrogenic origin (Ommayya 
classification). ${ }^{1}$ Although it is an effective procedure, overdrainage is associated with several serious and fatal complications.

Multiple factors dictate these complications and the rate of occurrence which include:

1. Number of attempts.

2. Size of the needle.

3. A spinal level where the tap is attempted.

Common adverse effects observed are infections, ${ }^{2}$ neurological injury from spinal drain insertion, ${ }^{3}$ pneumocephalus, ${ }^{4}$ epidural haematomas and CSF over drainage ${ }^{3,5}$ and associated complications. A study by Grady et al reviewing 530 consecutive lumbar drains for trans-sphenoidal surgery reports an incidence of $2.5 \%$ for postural headache related to over drainage, only $1.3 \%$ of them required an epidural blood patch. ${ }^{5}$ Minor symptoms include postural headache, VIth nerve palsy (as a sign of tight posterior fossa), visual obscuration, central herniation and decreased level of consciousness. ${ }^{3,6}$ Most of these aforementioned complications can be alleviated with cessation of the lumbar drain. ${ }^{3}$ Reports of patients being symptomatic 3 days after removal of lumbar drain, with bed rest, have been documented. In our case, he showed rapid deterioration within hours after lumbar drain placement.

Tonsillar herniation is a well-documented complication following lumbar drainage and has been noted in literature. ${ }^{7-10}$ However, acute tonsillar herniation after lumbar drainage is rare. The possible explanation behind the tonsillar descent is partly explained by the procedure itself and spinal fluid over drainage. It is also due to a change in the CSF dynamics in the posterior fossa and the pressure difference thence created between the intracranial and sub-occipital compartments. In an alert patient, neurological obtundation and presence or worsening of headache would be the hallmark of a tonsillar descent and central herniation. Early diagnosis and intervention in patients with high clinical suspicion should be advocated. Surgical treatment includes suboccipital craniectomy and decompression with lax duroplasty and $\mathrm{C} 1$ arch excision. Early intervention usually has a good neurological outcome and patients have improved functional outcome.

\section{Acknowledgments}

None.

\section{Conflicts of interest}

None.

\section{References}

1. Tolley NS, Brookes GB. Surgical management of cerebrospinal fluid rhinorrhoea. J R Coll Surg Edinb. 37(1):12-15.

2. Kitchel S, Eismont F, Green B. Closed subarachnoid drainage for management of cerebrospinal fluid leakage after an operation on the spine. J Bone Joint Surg. 1989;71A:984-987.

3. Roland PS, Marple BF, Meyerhoff WL, et al. Complication of lumbar spinal fluid drainage. Otolaryngol Head Neck Surg. 1992;107:564-569.

4. Graf C, Gross C, Bech D. Complications of spinal drainage in the management of cerebrospinal fluid leakage after operation on the spine. J Neurosurg. 1981;34:392-395.

5. Grady RE, Horlocker TT, Brown RD, et al. Neurologic complications afterplacement of cerebrospinal fluid drainage catheters and needles inanesthetized patients: implications for regional anethesia. Anesth Analg. 1999;88(2):388-392.

6. Pleasure SJ, Abosch A, Friedman J, et al. Spontaneous intracranial hypotension resulting in stupor caused by diencephalic compression. Neurology. 1998;50(6):1854-1857.

7. O’Shaughnessy BA, Bendok BR, Parkinson RJ, et al. Acquired Chiari malformation Type I associated with a supratentorialarteriovenous malformation. Case report and review of the literature. $J$ Neurosurg. 2006;104:28-32

8. Padmanabhan R, Crompton D, Burn D, et al. Acquired Chiari 1 malformation and syringomyelia following lumboperitoneal shunting for pseudotumourcerebri. J Neurol Neurosurg Psychiatry. 2005;76:298.

9. Sathi S, Stieg PE. "Acquired" Chiari I malformation after multiple lumbar punctures: case report. Neurosurgery. 1993;32:306-309.

10. Tubbs RS, Law C, Oakes WJ, et al. Acquired Chiari I malformation following baclofen pump placement in a child. Case report. J Neurosurg. 2004;101:211-213. 